\title{
Microfluidics as an Emerging Platform for Exploring Soil Environmental Processes: A Critical Review
}

Xiangyu Zhu ${ }^{1,2}$, Kun Wang ${ }^{1,2}$, Huicong Yan ${ }^{1,2}$, Congcong Liu ${ }^{1,2}$, Xiaoying Zhu ${ }^{1,2}$, Baoliang Chen ${ }^{1,2^{*}}$

${ }^{1}$ Department of Environmental Science, Zhejiang University, Hangzhou, Zhejiang 310058, China

${ }^{2}$ Zhejiang Provincial Key Laboratory of Organic Pollution Process and Control, Hangzhou, Zhejiang 310058, China

*Corresponding Author: Dr. Baoliang Chen

(Telephone \& Fax: +86-571-8898-2587, E-mail: blchen@,zju.edu.cn)

Number of pages: 9

Number of figures: 1

Number of tables: 2 


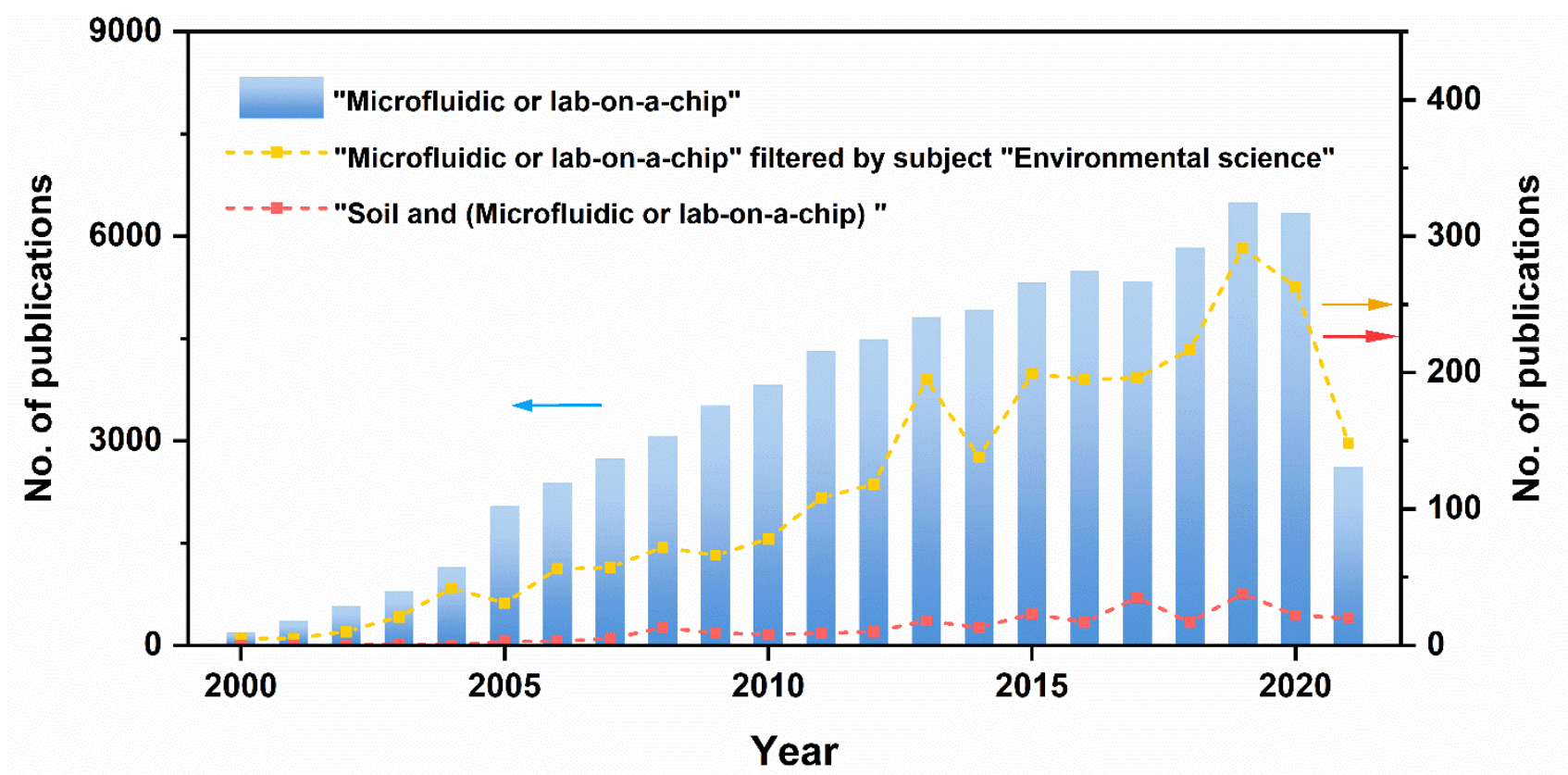

Figure S1. Annual number of publications on microfluidics and its applications in the field of environmental science and soil science (2000-2021). (Searched in Scopus database, 05/31/2021) 
Table S1. List of reviews on applications of microfluidics in environmental science and engineering

\begin{tabular}{lll}
\hline Year & Title & Ref. \\
\hline
\end{tabular}

General reviews

2019 A review of state-of-the-art microfluidic technologies for environmental applications: Detection and remediation

2020 Microfluidics for environmental applications

\section{Microfluidics as analytical devices for environmental monitoring}

2004 Lab-on-a-chip systems for biomedical and environmental monitoring 3

2005 Microfluidic devices for environmental monitoring

2005 Environmental microbiology-on-a-chip and its future impacts 5

2009 Applications of microfluidic systems in environmental analysis 6

2011 State-of-the-art lab chip sensors for environmental water monitoring 7

2012 Advances in microfluidics for environmental analysis 8

2012 Detection of Cryptosporidium in miniaturised fluidic devices 9

2013 Applications of autonomous microfluidic systems in environmental monitoring 10

2014 Application of microfluidics in waterborne pathogen monitoring: A review 11

2015 Trends in microfluidic systems for in situ chemical analysis of natural waters 12

2015 Detection of trace arsenic in drinking water: Challenges and opportunities for microfluidics 13

2016 Paper-based analytical devices for environmental analysis 14

2017 Microfluidic lab-on-a-chip platforms for environmental monitoring 15

2018 Lab-on-a-chip technology for environmental monitoring of microorganisms 16

2018 A review of centrifugal microfluidics in environmental monitoring 17

2018 Biotoxin sensing in food and environment via microchip 18

2019 Microfluidic paper-based analytical devices for environmental analysis of soil, air, ecology 19 and river water

2019 Paper-based analytical devices in environmental applications and their integration with portable technologies

2021 Lab-on-a-chip technology for in situ combined observations in oceanography 21

\section{Microfluidics as tools for toxicological studies}

2017 Organ-on-a-chip for assessing environmental toxicants 22

2018 Ecotoxicology goes on a chip: Embracing miniaturized bioanalysis in aquatic risk assessment 23

2021 Environmental toxicology wars: Organ-on-a-chip for assessing the toxicity of environmental 24 pollutants 


\begin{tabular}{clc}
\hline Year & \multicolumn{1}{c}{ Title } & Ref. \\
\hline 2021 & Emerging prospects of integrated bioanalytical systems in neuro-behavioral toxicology & 25 \\
2021 & $\begin{array}{l}\text { Emerging applications of microfluidic techniques for in vitro toxicity studies of atmospheric } \\
\end{array}$ & 26 \\
particulate matter &
\end{tabular}

\section{Microfluidics as microreactors for pollutant transformation, extraction, and materials production}

2014 Microfluidic reactors for photocatalytic water purification 27

2015 Nano-photocatalysts in microfluidics, energy conversion and environmental applications 28

2016 Microfluidic-based photocatalytic microreactor for environmental application: A review of 29 fabrication substrates and techniques, and operating parameters

2019 Microextractors applied in nuclear-spent fuel reprocessing: Micro/mini plants and 30 radiochemical analysis

\section{Microfluidics as platforms for environmental processes investigation}

2016 Soil-on-a-chip: Microfluidic platforms for environmental organismal studies 31

2018 Build your own soil: Exploring microfluidics to create microbial habitat structures 32

2018 Microfluidic model porous media: Fabrication and applications 33

2019 Application of microfluidic pore models for flow, transport, and reaction in geological porous 34 media: From a single test bed to multifunction real-time analysis tool

2020 Functionalized multiscale visual models to unravel flow and transport physics in porous 35 structures

2020 Review of microfluidic devices and imaging techniques for fluid flow study in porous 36 geomaterials

2021 Shining new light into soil systems: Spectroscopy in microfluidic soil chips reveals microbial 37 biogeochemistry

2021 Zooming in to acquire micro-reaction: Application of microfluidics on soil microbiome 
Table S2. Overview of different types of microfluidic devices

\begin{tabular}{|c|c|c|c|c|}
\hline $\begin{array}{l}\text { Type of } \\
\text { devices }\end{array}$ & $\begin{array}{l}\text { Common } \\
\text { fabrication } \\
\text { techniques }\end{array}$ & Advantages & Disadvantages & $\begin{array}{l}\text { Preferred application areas in } \\
\text { exploring soil processes }\end{array}$ \\
\hline Silicon-based & Photolithography & $\begin{array}{l}\text { Good chemical resistance, solvent } \\
\text { compatibility, and thermostability; } \\
\text { stable surface chemistry, well- } \\
\text { developed silicon surface } \\
\text { chemistry, low nonspecific } \\
\text { adsorption; resolution down to } \\
\text { nanometers; good reusability }\end{array}$ & $\begin{array}{l}\text { Fabrication requires cleanroom facility, } \\
\text { high-cost and time-consuming; non- } \\
\text { permeable to gases; opaque } \\
\text { (microscopy observation possible } \\
\text { when bonded with polymer or glass); } \\
\text { clogging }\end{array}$ & $\begin{array}{l}\text { Representation of nanometer-sized } \\
\text { geometries (e.g., soil nanopores); } \\
\text { dealing with organic chemicals } \\
\text { and solvents; dealing with high } \\
\text { pressure }\end{array}$ \\
\hline Glass-based & Photolithography & $\begin{array}{l}\text { Good chemical resistance, solvent } \\
\text { compatibility, and thermostability; } \\
\text { optically transparent; stable surface } \\
\text { chemistry, low nonspecific } \\
\text { adsorption; good reusability }\end{array}$ & $\begin{array}{l}\text { Fabrication requires cleanroom facility, } \\
\text { high-cost and time-consuming; non- } \\
\text { permeable to gases; fragile; clogging }\end{array}$ & $\begin{array}{l}\text { Microscopy observation; dealing } \\
\text { with organic chemicals and } \\
\text { solvents; dealing with high } \\
\text { pressure; integration with } \\
\text { electrochemical units; } \\
\text { electrophoresis }\end{array}$ \\
\hline PDMS-based (a) & Soft lithography & $\begin{array}{l}\text { Good optical transparency and gas } \\
\text { permeability; } \\
\text { relatively low-cost, allow rapid } \\
\text { prototyping and disposable use }\end{array}$ & $\begin{array}{l}\text { Low solvent compatibility; } \\
\text { hydrophobic surface, surface charge } \\
\text { not stable, typically require surface } \\
\text { modification; high nonspecific } \\
\text { adsorption }\end{array}$ & $\begin{array}{l}\text { Microscopy observation; long-term } \\
\text { cell culture; complex fluid control } \\
\text { using pumps and valves }\end{array}$ \\
\hline
\end{tabular}




\begin{tabular}{|c|c|c|c|c|}
\hline $\begin{array}{l}\text { Type of } \\
\text { devices }\end{array}$ & $\begin{array}{l}\text { Common } \\
\text { fabrication } \\
\text { techniques }\end{array}$ & Advantages & Disadvantages & $\begin{array}{l}\text { Preferred application areas in } \\
\text { exploring soil processes }\end{array}$ \\
\hline $\begin{array}{l}\text { Thermoplastics- } \\
\text { based }^{(b)}\end{array}$ & Thermomolding & $\begin{array}{l}\text { Good optical transparency; allow } \\
\text { large-scale production and } \\
\text { commercialization }\end{array}$ & $\begin{array}{l}\text { Relatively low solvent compatibility } \\
\text { (better than PDMS); non-permeable } \\
\text { to gases; hydrophobic surface; } \\
\text { fragile; relatively low resolution in } \\
\text { microfabrication }\end{array}$ & $\begin{array}{l}\text { Microscopy observation; integration } \\
\text { with electrochemical units; } \\
\text { millifluidic devices }\end{array}$ \\
\hline Hydrogel-based & $\begin{array}{l}\text { Casting, } \\
\text { photopolymerization }\end{array}$ & $\begin{array}{l}\text { High permeability; allow } \\
\text { functionalization with stimuli- } \\
\text { responsive behavior }\end{array}$ & $\begin{array}{l}\text { Low resolution in microfabrication; } \\
\text { difficulty with bonding }\end{array}$ & $\begin{array}{l}\text { Embedding cells for } 3 \mathrm{D} \text { culture; } \\
\text { controlling fluid flows or creating } \\
\text { concentration gradients }\end{array}$ \\
\hline Paper-based & $\begin{array}{l}\text { Printing, } \\
\text { photolithography }\end{array}$ & $\begin{array}{l}\text { Substrate materials cheap and readily } \\
\text { available; easy fabrication; } \\
\text { disposable; functional integration } \\
\text { of flow, filtering, and separation }\end{array}$ & $\begin{array}{l}\text { Sensitivity and selectivity for detection } \\
\text { may be insufficient }\end{array}$ & $\begin{array}{l}\text { Portable soil analysis with } \\
\text { colorimetric and electrochemical } \\
\text { detection }\end{array}$ \\
\hline 3D-printed & $\begin{array}{l}\text { Stereolithography, } \\
\text { fused deposition } \\
\text { modeling }\end{array}$ & $\begin{array}{l}\text { No requirement of cleanroom facility; } \\
\text { relatively low-cost; allow rapid } \\
\text { prototyping }\end{array}$ & $\begin{array}{l}\text { Low resolution in microfabrication } \\
\text { (typically at several hundred } \\
\text { micrometers); microscopy } \\
\text { observation challenging }\end{array}$ & $\begin{array}{l}\text { Rapidly fabricating proof-of-concept } \\
\text { devices for testing; create truly 3D } \\
\text { architectures (soil micromodels); } \\
\text { millifluidic devices }\end{array}$ \\
\hline
\end{tabular}

Notes: (a) PDMS, polydimethylsiloxane.

(b) Common thermoplastics for microfluidics include polycarbonate (PC), poly(methyl methacrylate) (PMMA), cyclic-olefin copolymer (COC), polystyrene (PS). 


\section{References}

(1) Yew, M.; Ren, Y.; Koh, K. S.; Sun, C.; Snape, C. A Review of State-of-the-Art Microfluidic Technologies for Environmental Applications: Detection and Remediation. Glob. Chall. 2019, 3 (1), 1800060.

(2) Wang, T.; Yu, C.; Xie, X. Microfluidics for Environmental Applications. In Advances in Biochemical Engineering/Biotechnology; Springer Berlin Heidelberg, 2020.

(3) Gardeniers, J. G. E.; van den Berg, A. Lab-on-a-Chip Systems for Biomedical and Environmental Monitoring. Anal. Bioanal. Chem. 2004, 378 (7), 1700-1703.

(4) Marle, L.; Greenway, G. M. Microfluidic Devices for Environmental Monitoring. TrAC Trends Anal. Chem. 2005, 24 (9), 795-802.

(5) Liu, W.-T.; Zhu, L. Environmental Microbiology-on-a-Chip and Its Future Impacts. Trends Biotechnol. 2005, 23 (4), 174-179.

(6) Li, H.-F.; Lin, J.-M. Applications of Microfluidic Systems in Environmental Analysis. Anal. Bioanal. Chem. 2009, 393 (2), 555-567.

(7) Jang, A.; Zou, Z.; Lee, K. K.; Ahn, C. H.; Bishop, P. L. State-of-the-Art Lab Chip Sensors for Environmental Water Monitoring. Meas. Sci. Technol. 2011, 22 (3), 032001.

(8) Jokerst, J. C.; Emory, J. M.; Henry, C. S. Advances in Microfluidics for Environmental Analysis. Analyst 2012, 137 (1), 24-34.

(9) Bridle, H.; Kersaudy-Kerhoas, M.; Miller, B.; Gavriilidou, D.; Katzer, F.; Innes, E. A.; Desmulliez, M. P. Y. Detection of Cryptosporidium in Miniaturised Fluidic Devices. Water Res. 2012, 46 (6), 1641-1661.

(10) Campos, C. D. M.; da Silva, J. A. F. Applications of Autonomous Microfluidic Systems in Environmental Monitoring. RSC Adv. 2013, 3 (40), 18216.

(11) Bridle, H.; Miller, B.; Desmulliez, M. P. Y. Application of Microfluidics in Waterborne Pathogen Monitoring: A Review. Water Res. 2014, 55, 256-271.

(12) Nightingale, A. M.; Beaton, A. D.; Mowlem, M. C. Trends in Microfluidic Systems for in Situ Chemical Analysis of Natural Waters. Sens. Actuators B Chem. 2015, 221, 1398-1405.

(13) Yogarajah, N.; Tsai, S. S. H. Detection of Trace Arsenic in Drinking Water: Challenges and Opportunities for Microfluidics. Environ. Sci. Water Res. Technol. 2015, 1 (4), 426-447.

(14) Meredith, N. A.; Quinn, C.; Cate, D. M.; Reilly, T. H.; Volckens, J.; Henry, C. S. Paper-Based Analytical Devices for Environmental Analysis. Analyst 2016, 141 (6), 1874-1887.

(15) Pol, R.; Céspedes, F.; Gabriel, D.; Baeza, M. Microfluidic Lab-on-a-Chip Platforms for Environmental Monitoring. TrAC Trends Anal. Chem. 2017, 95, 62-68.

(16) Dhar, B. C.; Lee, N. Y. Lab-on-a-Chip Technology for Environmental Monitoring of Microorganisms. BioChip J. 2018, 12 (3), 173-183.

(17) Maguire, I.; O’Kennedy, R.; Ducrée, J.; Regan, F. A Review of Centrifugal Microfluidics in Environmental Monitoring. Anal. Methods 2018, 10 (13), 1497-1515.

(18) Zhang, Z.; Yu, L.; Xu, L.; Hu, X.; Li, P.; Zhang, Q.; Ding, X.; Feng, X. Biotoxin Sensing in Food and Environment via Microchip. Electrophoresis 2014, 35 (11), 1547-1559.

(19) Kung, C.-T.; Hou, C.-Y.; Wang, Y.-N.; Fu, L.-M. Microfluidic Paper-Based Analytical Devices for Environmental Analysis of Soil, Air, Ecology and River Water. Sens. Actuators B Chem. 2019, 301, 126855. 
(20) Marquez, S.; Liu, J.; Morales-Narváez, E. Paper-Based Analytical Devices in Environmental Applications and Their Integration with Portable Technologies. Curr. Opin. Environ. Sci. Health 2019, 10, 1-8.

(21) Fukuba, T.; Fujii, T. Lab-on-a-Chip Technology for in Situ Combined Observations in Oceanography. Lab. Chip 2021, 21 (1), 55-74.

(22) Cho, S.; Yoon, J.-Y. Organ-on-a-Chip for Assessing Environmental Toxicants. Curr. Opin. Biotechnol. 2017, 45, 34-42.

(23) Campana, O.; Wlodkowic, D. Ecotoxicology Goes on a Chip: Embracing Miniaturized Bioanalysis in Aquatic Risk Assessment. Environ. Sci. Technol. 2018, 52 (3), 932-946.

(24) Yang, S.; Chen, Z.; Cheng, Y.; Liu, T.; Lihong Yin; Pu, Y.; Liang, G. Environmental Toxicology Wars: Organ-on-a-Chip for Assessing the Toxicity of Environmental Pollutants. Environ. Pollut. 2021, 268, 115861.

(25) Bai, Y.; Henry, J.; Campana, O.; Wlodkowic, D. Emerging Prospects of Integrated Bioanalytical Systems in Neuro-Behavioral Toxicology. Sci. Total Environ. 2021, 756, 143922.

(26) Liu, F.; Ng, N. L.; Lu, H. Emerging Applications of Microfluidic Techniques for in Vitro Toxicity Studies of Atmospheric Particulate Matter. Aerosol Sci. Technol. 2021, 1-17.

(27) Wang, N.; Zhang, X.; Wang, Y.; Yu, W.; Chan, H. L. W. Microfluidic Reactors for Photocatalytic Water Purification. Lab. Chip 2014, 14 (6), 1074-1082.

(28) Parmar, J.; Jang, S.; Soler, L.; Kim, D.-P.; Sánchez, S. Nano-Photocatalysts in Microfluidics, Energy Conversion and Environmental Applications. Lab. Chip 2015, 15 (11), 2352-2356.

(29) Das, S.; Srivastava, V. C. Microfluidic-Based Photocatalytic Microreactor for Environmental Application: A Review of Fabrication Substrates and Techniques, and Operating Parameters. Photochem. Photobiol. Sci. 2016, 15 (6), 714-730.

(30) Wang, T.; Xie, T.; Xu, C. Microextractors Applied in Nuclear-Spent Fuel Reprocessing: Micro/Mini Plants and Radiochemical Analysis. Crit. Rev. Environ. Sci. Technol. 2019, 49 (1), $1-31$.

(31) Stanley, C. E.; Grossmann, G.; Solvas, X. C. i; deMello, A. J. Soil-on-a-Chip: Microfluidic Platforms for Environmental Organismal Studies. Lab. Chip 2016, 16 (2), 228-241.

(32) Aleklett, K.; Kiers, E. T.; Ohlsson, P.; Shimizu, T. S.; Caldas, V. E.; Hammer, E. C. Build Your Own Soil: Exploring Microfluidics to Create Microbial Habitat Structures. ISME J. 2018, 12 (2), 312-319.

(33) Anbari, A.; Chien, H.-T.; Datta, S. S.; Deng, W.; Weitz, D. A.; Fan, J. Microfluidic Model Porous Media: Fabrication and Applications. Small 2018, 14 (18), 1703575.

(34) Cao, S. C.; Jung, J.; Radonjic, M. Application of Microfluidic Pore Models for Flow, Transport, and Reaction in Geological Porous Media: From a Single Test Bed to Multifunction Real-Time Analysis Tool. Microsyst. Technol. 2019, 25 (11), 4035-4052.

(35) Zhang, Y.; Khorshidian, H.; Mohammadi, M.; Sanati-Nezhad, A.; Hejazi, S. H. Functionalized Multiscale Visual Models to Unravel Flow and Transport Physics in Porous Structures. Water Res. 2020, 175, 115676.

(36) Jahanbakhsh, A.; Wlodarczyk, K. L.; Hand, D. P.; Maier, R. R. J.; Maroto-Valer, M. M. Review of Microfluidic Devices and Imaging Techniques for Fluid Flow Study in Porous Geomaterials. Sensors 2020, 20 (14), 4030. 
(37) Pucetaite, M.; Ohlsson, P.; Persson, P.; Hammer, E. Shining New Light into Soil Systems: Spectroscopy in Microfluidic Soil Chips Reveals Microbial Biogeochemistry. Soil Biol. Biochem. 2021, 153, 108078.

(38) Zhang, X.; Wu, S.; Sun, X.; Mortimer, M.; Wu, Y.; Zhang, M.; Huang, Q.; Cai, P. Zooming in to Acquire Micro-Reaction: Application of Microfluidics on Soil Microbiome. Soil Ecol. Lett. 2021, https://doi.org/10.1007/s42832-021-0073-7. 